\title{
COMPARAÇÃO DO USO DO LASER DE BAIXA POTÊNCIA (660 NM E 830 NM) EM ESTRIAS ATRÓFICAS DE MULHERES JOVENS
}

\section{Amanda Kruger}

Universidade Estadual do Oeste do Paraná - Unioeste, Brasil.

Ana Beatriz Campanhoni Amadori

Universidade Estadual do Oeste do Paraná - Unioeste, Brasil.

\section{Claudia Caroline Vergutz}

Universidade Estadual do Oeste do Paraná - Unioeste, Brasil.

\section{Nathyelle Cristiane Golyjewski Vilaca}

Universidade Estadual do Oeste do Paraná - Unioeste, Brasil.

\section{Patricia Regina Hickmann}

Universidade Estadual do Oeste do Paraná - Unioeste, Brasil.

Celeide Pinto Aguiar Peres

Universidade Estadual do Oeste do Paraná - Unioeste, Brasil.

\section{Gladson Ricardo Flor Bertolini}

Laboratório de Estudo das Lesões e Recursos Fisioterapêuticos da Universidade Estadual do Oeste do Paraná - Unioeste, Brasil.
Autor correspondente

Gladson Bertolini

gladsonricardo@gmail.com
RESUMO: O laser de baixa potência é um recurso utilizado em lesões de pele, contudo ainda pouco explorado no tratamento de estrias alba. Objetivo: comparar dois comprimentos de laser de baixa potência ( 660 $\mathrm{nm}$ e $830 \mathrm{~nm}$ ) sobre estrias albas. Um grupo de 24 mulheres foi dividido em três: G1 - $660 \mathrm{~nm}$; G2 - $830 \mathrm{~nm}$; G3 - placebo. Realizaram-se 12 aplicações de laser, durante quatro semanas. Foram avaliadas fotos das estrias no antes, depois e após duas semanas das aplicações do laser para mensurar a área ocupada pelas estrias por meio de planimetria digitalizada, bem como seus aspectos. Pela planimetria observou-se redução estatisticamente significativa na área ocupada em G1. Na análise qualitativa, o grupo tratado com laser de $660 \mathrm{~nm}$ apresentou melhora no aspecto das estrias. A aplicação utilizando o laser de baixa potência com comprimento de onda de $660 \mathrm{~nm}$ demonstrou efeitos positivos na área analisada, bem como no aspecto das estrias.

PALAVRAS-CHAVE: Estrias de distensão; Terapia com luz de baixa intensidade; Pele.

\section{COMPARING LOW POWER LASER USE (660 NM AND 830 NM) IN ATROPHIC STRIAE IN YOUNG FEMALES}

ABSTRACT: Although low-power laser is employed in skin lesions, it is only scantily used in the treatment of white striae. Comparison of two low-power laser lengths $(660 \mathrm{~nm}$ and $830 \mathrm{~nm})$ on white striae. A group of 24 females was subdivided into three groups: $\mathrm{G} 1-660 \mathrm{~nm}$; G2 - $830 \mathrm{~nm}$; G3 - placebo. Twelve laser applications were undertaken during four weeks. Photographs of striae before, after and after two weeks of laser application were performed to measure area with striae by digital planimetry and their aspects. Planimetry revealed statistically significant decrease in area occupied in G1. Group treated with 660 $\mathrm{nm}$ laser qualitatively improved striae aspect. Application with lower-power laser at $660 \mathrm{~nm}$ had a positive effect on the area analyzed and in the aspects of the striae.

KEY WORDS: Distension striae; Low intensity laser therapy; Skin.

\section{INTRODUÇÃO}

A pele é o maior órgão do corpo humano, com extensão de $15 \%$ do total corporal, e é definida como um tecido de origem endodérmico, composta por três camadas distintas: epiderme, derme e hipoderme; formando uma barreira contra agressões exógenas e impede a passagem da água e proteínas para o meio externo. Funciona como um órgão sensorial e participa do sistema imunológico, sendo 
responsável pela proteção, regulação de temperatura e sensibilidade do organismo ${ }^{1,2}$.

As estrias de distensão são lesões dérmicas permanentes comuns que ocorrem comumente neste órgão. Surgem em áreas de estiramento dérmico, e sua ocorrência é mais comum em abdômen, seios, nádegas e coxas. Influências hormonais, redução de fibronectina, colágeno e elastina, além do alongamento mecânico sobre a pele, são fatores responsáveis na formação de estrias. $\mathrm{Na}$ forma aguda são de aspecto vermelho/violácea (estrias rubras) e na forma crônica (estria alba) formam depressão dérmica hipopigmentada. Por causa de sua alta prevalência e impacto sobre a qualidade de vida, principalmente em mulheres, há grande demanda para um tratamento efetivo. Embora sua completa erradicação não é atingível, é possível melhora na sua aparência, reduzindo sintomas físicos e psicológicos, por meio do aumento da produção de colágeno, redução de eritema ou aumento da pigmentaçãa $0^{3-6}$.

Dentre os tratamentos comumente utilizados, pode-se citar o uso de agentes tópicos (azeite de oliva, ácido boswellic, extrato de cebola, manteiga de cacau, centella asiática etc.), microgalvanopuntura, radiofrequência e fototerapia, porém, cientificamente comprovada sua efetividade se dá por aplicação da radiofrequência e fototerapia. Dentre estes, destacam-se a luz intensa pulsada e de laser, que podem ser de alta ou baixa potência. O laser de alta potência tem efeito ablativo, removendo a epiderme e provocando danos térmicos à derme, necessitando de tempo de recuperação prolongado e promovendo danos colaterais (como hiperpigmentação). Para minimizar tais efeitos, a aplicação do laser fracionado com intervalos entre os trens de pulso gera menor aquecimento tecidual ${ }^{4,7-9}$.

$\mathrm{Na}$ fisioterapia a utilização do laser de baixa potência e diodo emissor de luz (light-emitting diode - LED) tem apresentado resultados importantes em lesões da pele, com redução na intensidade de resposta inflamatória, promovendo melhor organização das fibras colágenas, aumento da neovascularização e da proliferação fibroblástica, com resposta satisfatória no reparo tecidual $^{10-13}$ principalmente em estrias de distensão com a utilização de laser de baixas fluências ${ }^{14-16}$

Para o laser de baixa potência, parâmetros como potência, comprimento de onda, tempo de irradiação, fluência e irradiância podem diversificar os resultados ${ }^{17}$. O objetivo do presente estudo foi comparar os efeitos da aplicação do laser de baixa potência de dois comprimentos de onda distintos (660 nm e $830 \mathrm{~nm})$ em estrias albas na região lateral da coxa em mulheres jovens.

\section{METODOLOGIA}

Este estudo é classificado como experimental, transversal, randomizado, realizado entre junho e novembro de 2018, no Centro de Reabilitação Física da Universidade Estadual do Oeste do Paraná (Unioeste), na cidade de Cascavel - Paraná. A pesquisa foi aprovada pelo Comitê de Ética em Humanos da Unioeste (parecer consubstanciado 2.748.119). Os participantes assinaram o Termo de Consentimento Livre e Esclarecido indicando que estavam cientes em participar do presente estudo.

A amostra foi composta por 24 indivíduos do sexo feminino, acadêmicas da graduação em fisioterapia da Unioeste. Os critérios de inclusão foram: sexo feminino com idade entre 18 e 28 anos que possuíam estrias albas na região lateral da coxa bilateral. Os critérios de exclusão foram: 1) estar realizando ou já ter realizado outro tipo de tratamento para estrias; 2) estado gravídico; 3) uso de medicamentos esteroides; 4) tabagismo; 5) diagnóstico de câncer; 6) presença de doenças hemorrágicas; 7) diagnóstico de epilepsia ou trombose; e 8) alteração cognitiva.

Realizou-se um sorteio com o nome das participantes em envelope opaco, para alocá-las em três grupos, contendo oito participantes em cada grupo. Para o G1: estabeleceu-se a intervenção com o laser $660 \mathrm{~nm}$; G2: a intervenção com o laser de $830 \mathrm{~nm}$; e G3: o grupo placebo. O grupo controle (GC) foi composto pela mesma amostra, levando em consideração a região homóloga do membro pélvico contralateral, a qual não teria a intervenção do laser. Durante a pesquisa, a amostra foi orientada a não receber qualquer outro tipo de intervenção nas estriais em estudo. Os dados pessoais de cada participante como idade, massa corporal, estatura, circunferência do quadril e coxa, cor de pele, ano de menarca, cor das estrias, sensibilidade dolorosa na região, 
número de gestações e presença ou não de patologias dérmicas, transtornos circulatórios ou de cicatrização, foram obtidos através de um questionário no início da pesquisa.

\section{PROTOCOLO DE INTERVENÇÃO}

Utilizou-se um gabarito produzido em papel sulfite $(10 \times 10 \mathrm{~cm})$, com 36 perfurações que permitiam a passagem da ponta de uma caneta a uma distância de 1 cm entre cada perfuração. Marcou-se, através dessas perfurações, com caneta permanente, a pele da região lateral da coxa esquerda de cada participante. Em cada marcação, aplicou-se o laser durante quatro semanas, com três intervenções semanais, totalizando 12 aplicações. O aparelho utilizado no estudo foi o Laserpulse Ibramed ${ }^{\circledR}$ de diodo, com comprimentos de onda de $660 \mathrm{~nm}$ e $830 \mathrm{~nm}$, ambos com potência de $30 \mathrm{~mW}$, densidade de energia de $4 \mathrm{~J} / \mathrm{cm}^{2}$ e energia total de de $8,64 \mathrm{~J}$ utilizando a caneta de $660 \mathrm{~nm}$ e $17,28 \mathrm{~J}$ com a caneta de $830 \mathrm{~nm}$.

A aplicação foi pontual, em 36 pontos, e as canetas lasers foram posicionadas em $90^{\circ}$ diretamente com a pele, com duração de 8 segundos em cada ponto a aplicação da caneta laser de $660 \mathrm{~nm}$ no grupo G1 e 16 segundos a aplicação da caneta laser de $830 \mathrm{~nm}$. No G3, foram realizados os mesmos procedimentos dos grupos G1 e G2, porém o aparelho, apesar de ligado, não emitia radiação, ou seja, as canetas lasers não foram ativadas. Antes de dar início à intervenção, ambas as canetas lasers foram calibradas.

As participantes se posicionaram em decúbito lateral direito, mantendo a área lateral da coxa desnuda para a aplicação da caneta laser. A região da pele da coxa, as canetas lasers e a maca utilizada, para o posicionamento das participantes, foi higienizada com algodão embebido em álcool 70\%. Todas as participantes usaram óculos de proteção contra as irradiações do laser.
AVALIAÇÕES

No início e final das intervenções a amostra foi submetida a coleta a massa corporal, estatura e circunferência da coxa (Filizola eebeyond technology ${ }^{\circledR}, 0$, 200 $\mathrm{kg}$ ). Foi calculado o IMC (Índice de Massa Corpórea) para descartar possíveis influências de ganho ou perda de massa corporal sobre o aspecto da pele e das estrias, e análise da homogeneidade entre grupos. Fotografias das regiões estudadas foram realizadas para avaliação por planimetria digitalizada.

As fotografias foram registradas e avaliadas em três momentos. Primeira avaliação (AVA1, prévio à aplicação do laser) após 12 das intervenções, segunda avaliação (AVA2) e após duas semanas do término das intervenções e terceira avaliação (AVA3) do GC nestes mesmos momentos para análise dos aspectos das estrias. A participante era posicionada em decúbito lateral em uma maca com uma estrutura de madeira, com altura de $40 \mathrm{~cm}$. A câmera fotográfica digital Canon EOS Rebel T3 12.2 MP foi posicionada atrás da região que recebeu a aplicação do laser fixada em suporte. Todas os registros fotográficos foram realizados no mesmo ambiente com a mesma intensidade de luz.

Utilizou-se o programa Image-Pro Plus ${ }^{\circledR}$ para análise quantitativa das fotografias da área ocupada pelas estrias, por planimetria. Realizou-se a sobreposição de uma grade com 150 pontos, e a partir disso, o número de pontos que sobrepunham as estrias foram contabilizados. Utilizou-se a fórmula: número de pontos x 100/150, obtendo assim a porcentagem da área em que as estrias ocupavam. Os valores de cada grupo foram expressos em média e desvio padrão. Também foi realizada análise qualitativa das mesmas através da observação visual

\section{ANÁLISE ESTATÍSTICA}

Para realização da análise estatística foi utilizado o programa Bioestat 5.0, visto que não houve normalidade nos dados, avaliado por meio do teste de Shapiro-Wilk, os mesmos foram apresentados em mediana, primeiro e terceiro quartis. Para comparações intragrupo o teste de Friedman foi realizado. Para as comparações intergrupos utilizou-se o teste Kruskal-Wallis. Em todos os casos o nível de significância aceito foi de $5 \%(p<0,05)$. 


\section{RESULTADOS}

Na Tabela 1 observa-se que os três grupos: G1 (660nm), G2 (830nm) e G3 (placebo), foram homogêneos em relação aos critérios avaliados.

Tabela 1. Caracterização da amostra

\begin{tabular}{|l|c|c|c|}
\hline & G1 & G2 & G3 \\
\hline Idade (anos) & $19.5 \pm 1,2$ & $22.0 \pm 1.2$ & $20.0 \pm 1.4$ \\
\hline IMC - inicial $\left(\mathrm{kg} / \mathrm{m}^{2}\right)$ & $21.9 \pm 3.5$ & $23.7 \pm 3.0$ & $20.6 \pm 1.0$ \\
\hline IMC - final $\left(\mathrm{kg} / \mathrm{m}^{2}\right)$ & $19.5 \pm 1.2$ & $23.7 \pm 3.2$ & $20.7 \pm 1.0$ \\
\hline $\begin{array}{l}\text { Circunferência coxa - } \\
\text { inicial (cm) }\end{array}$ & $57.9 \pm 3.9$ & $60.6 \pm 6.2$ & $56.8 \pm 4.4$ \\
\hline $\begin{array}{l}\text { Circunferência coxa - } \\
\text { final (cm) }\end{array}$ & $58.4 \pm 4.3$ & $60.7 \pm 5.8$ & $57.0 \pm 2.8$ \\
\hline $\begin{array}{l}\text { Circunferência quadril } \\
\text {-inicial (cm) }\end{array}$ & $99.7 \pm 8.2$ & $104.7 \pm 6.7$ & $97.35 \pm 4.1$ \\
\hline $\begin{array}{l}\text { Circunferência quadril } \\
\text { - final (cm) }\end{array}$ & $99.7 \pm 7.5$ & $104.3 \pm 6.4$ & $97.6 \pm 4.1$ \\
\hline
\end{tabular}

A análise dos resultados pela planimetria demostrou que houve redução apenas nos valores percentuais da área ocupada pelas estrias na última avaliação (AVA3) quando comparados à primeira (AVA1). E com relação ao grupo controle (G3) comparado ao G1, onde foi aplicada a caneta laser de $660 \mathrm{~nm}$ (Tabela 2).

Tabela 2. Resultados da avaliação por planimetria digitalizada realizada em estrias albas de acordo com o grupo: G1(laser de 660nm), G2 (laser de 830nm), G3 (Placebo) e Controle (Lado contralateral da aplicação). Os dados são relativos à mediana e média dos ranks da porcentagem da área ocupada pelas estrias

\begin{tabular}{|l|l|l|l|l|l|}
\hline & AVA1 & AVA2 & AVA3 & Controle & p-valor \\
\hline G1 & $24.0-3.6 \mathrm{a}$ & $21.0-2.1 \mathrm{ab}$ & $20.0-1.2 \mathrm{~b}$ & $22.3-3.1 \mathrm{a}$ & 0,0009 \\
\hline G2 & $24.7-3.1$ & $22.7-2.5$ & $22.0-1.6$ & $22-2.7$ & 0,1708 \\
\hline G3 & $16.7-2.6$ & $16.7-2.4$ & $17.3-2.2$ & $17.3-2.8$ & 0,8358 \\
\hline p-valor & 0,7969 & 0,6287 & 0,6185 & 0,6533 & \\
\hline
\end{tabular}

Letras iguais apresentam semelhança estatística na comparação intragrupo para G1.

$\mathrm{Na}$ análise qualitativa, observando visualmente as fotografias realizadas na AVA1 e AVA3, foi possível observar melhora no aspecto das estrias quanto à tonalidade, ao tamanho e à largura das estrias, deixando a pele mais homogênea no G1 e G2. Com maior ênfase desses resultados na utilização do laser com o comprimento de onda de $660 \mathrm{~nm}$, os valores estão apresentados na Tabela 2.
Demostrando, assim, melhores efeitos na redução das estrias estudadas, como demostrado na Figura 1. Comparando os resultados nas AVA1 e AVA3, para os três grupos.

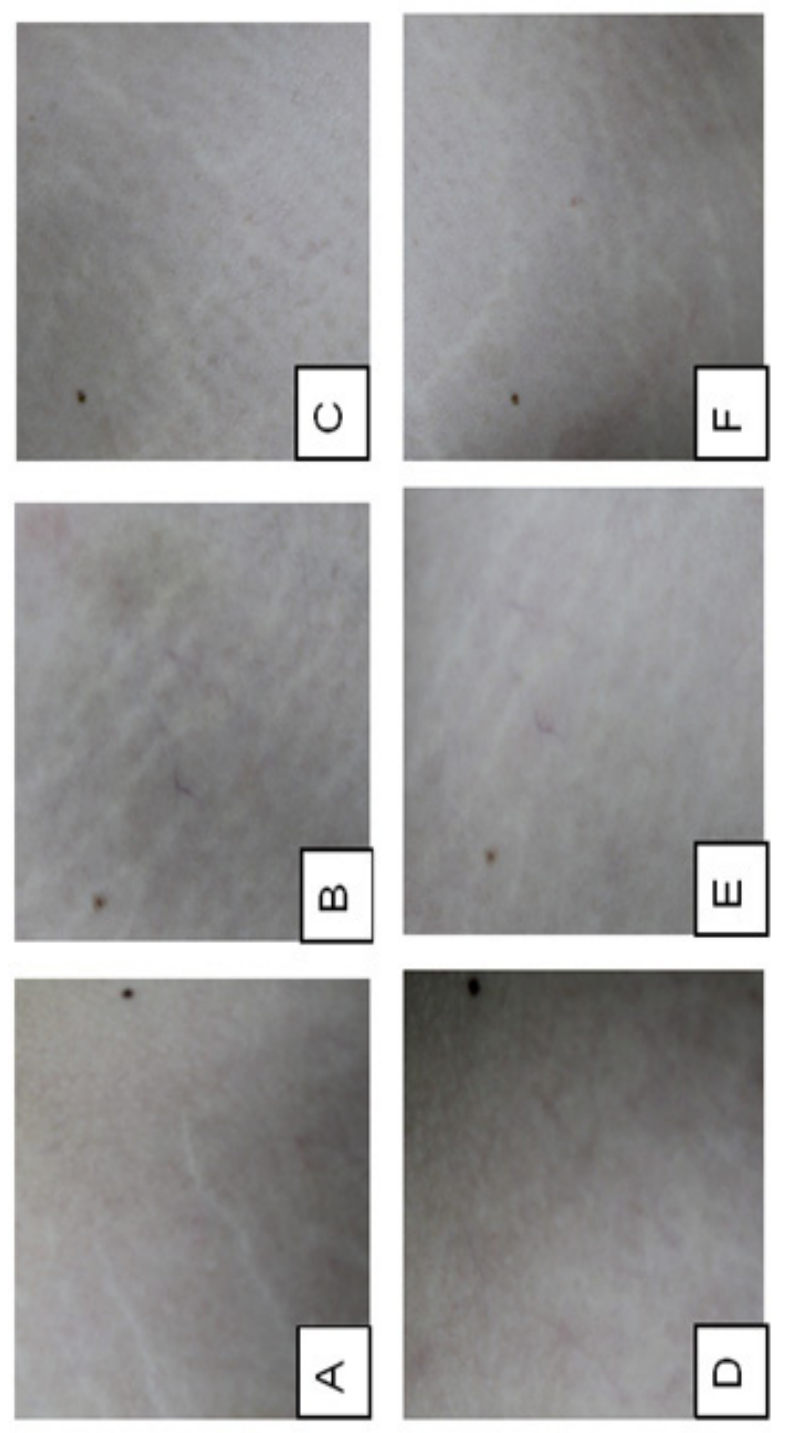

Figura 1. Representação fotográfica das imagens de G1 (A e D), G2 (B e E) e G3 (C e F) demostrando o aspecto das estrias no início das intervenções (A, B e C) e após duas semanas do término (D, E e F) 


\section{DISCUSSÃO}

O presente estudo apresentou alterações qualitativas e quantitativas (área) em estrias após tratamento com laser de baixa potência no comprimento de onda de $660 \mathrm{~nm}$, além de alterações qualitativas quando utilizado o de $830 \mathrm{~nm}$. Visto que o tratamento para estrias representa um desafio dermatológico ${ }^{4}$. A literatura apresenta o laser como uma útil terapia para o tratamento de estrias, com melhores resultados em estrias rubras, contudo os equipamentos utilizados são os lasers fracionários, que geralmente geram danos térmicos aos tecidos ${ }^{8}$. Desta forma torna-se interessante a realização de pesquisas que avaliem o laser de baixa potência, pois o mesmo pode apresentar importantes efeitos nas células epiteliais, pelo estímulo à proliferação e ao aumento na microcirculação ${ }^{18,19}$ part of Springer Nature Burn is defined as a traumatic injury of thermal origin, which affects the organic tissue. Low-level laser therapy (LLLT).

Maldaner et al. ${ }^{20}$ avaliaram o uso do laser de 660 $\mathrm{nm}$, em cultura de fibroblastos, e observaram que a fluência de $4 \mathrm{~J} / \mathrm{cm}^{2}$ produziu importantes efeitos, como aumento na proliferação, aumento de IL-10, FGF-1 e KGF, além de reversão no estado de oxidação do DNA e redução de interleucinas inflamatórias (IL-1B, IL-6 e INFy). Além destes efeitos, o laser de baixa potência tem apresentado efeitos angiogênicos e aumento em diversos fatores de crescimento importantes no reparo tecidual ${ }^{21,22}$, por tais efeitos vislumbram-se possibilidades de alterações em estrias atróficas por parte destes equipamentos.

A amostra relatou o aparecimento das estrias atróficas durante a puberdade, época que é muito comum o surgimento das mesmas, e optou-se avaliar a região lateral das coxas pela alta prevalência de estrias em mulheres nesta região. ${ }^{4,9}$ De acordo com o relato das participantes, inicialmente as estrias apresentaram-se características rubras, posteriormente amadureceram e se tornaram brancas e atróficas (estria alba) pela quebra e reorganização do colágeno e elastina. Desta forma propôs-se observar a ação da radiação laser de baixa potência, que provoca modificações bioquímicas, bioelétricas e bioenergéticas, atuando no aumento do metabolismo, na proliferação e maturação celular, na quantidade de tecido de granulação e na diminuição dos mediadores inflamatórios, induzindo o processo de cicatrização e consequentemente a síntese de colágeno ${ }^{23}$.

Os dados do presente estudo corroboram àqueles observados por Busata et al..$^{15}$, que tiveram o mesmo método de aplicação de 12 sessões com LBP de $4 \mathrm{~J} / \mathrm{cm}^{2} \mathrm{com}$ potência de $30 \mathrm{~mW}$, no qual o comprimento de onda de $660 \mathrm{~nm}$ mostrou-se um método não invasivo, indolor e de rápida aplicação, com efeitos positivos após quatro semanas das aplicações. Segundo Castano et al. ${ }^{17}$, diferenças em parâmetros de radiação, como fluência, irradiância, tempo de irradiação e comprimentos de onda, podem produzir diferentes resultados terapêuticos. Assim, neste estudo utilizaram-se fluências semelhantes para os equipamentos, tendo como forma de avaliação quantitativa a planimetria que apresentou resultados significativos apenas para o grupo $660 \mathrm{~nm}$, já para a avaliação qualitativa, que levou em consideração o aspecto da estria, ambos os comprimentos de onda se mostraram vantajosos, mas, novamente $660 \mathrm{~nm}$ apresentou melhores resultados.

Smith ${ }^{24}$ apresenta uma hipótese que o laser no comprimento de onda $660 \mathrm{~nm}$ ativa a célula via absorção no citocromo c?? oxidase, e desta forma produz uma cascata partindo das mitocôndrias, mas, que o laser no comprimento de onda $830 \mathrm{~nm}$ ativaria diretamente canais de cálcio na membrana celular, alterando a permeabilidade desta. Ainda, Bjordal et al. ${ }^{25}$ apontam que para comprimentos de onda menores a pele tem maior interferência como barreira; desta forma poderiam atuar com maior eficiência nesta região. Tais fatos podem explicar as diferenças encontradas no presente estudo entre G1 e $\mathrm{G} 2$, visto os melhores resultados obtidos pelo primeiro, $\mathrm{o}$ mesmo se apresenta mais indicado para aplicações clínicas em indivíduos com estrias atróficas, com o objetivo de reduzir as mesmas bem como a alteração de tonalidade, sendo esta uma aplicação prática da presente pesquisa.

Destaca-se como limitação deste estudo a dificuldade da análise da energia total entregue, bem como a análise do tempo de irradiação promovido nas intervenções. As intervenções foram realizadas em mais de um equipamento dificultando a análise desses parâmetros, visto que apesar de potências semelhantes os equipamentos possuem spots diferentes, ou seja, para semelhantes fluências o tempo de terapia torna-se diferente. Sugerem-se futuras pesquisas para estas análises. Sugere-se tam- 
bém que futuros estudos avaliem estrias atróficas, o uso de equipamentos do tipo cluster, que além da radiação com laser de baixa potência associam-se LEDs a este, o que na prática aumenta a energia entregue na região.

\section{CONCLUSÃO}

Concluiu-se que a utilização do laser de baixa potência com comprimento de onda de $660 \mathrm{~nm}$ obteve efeitos positivos na área das estrias albas desta amostra, bem como no aspecto das mesmas quanto à tonalidade, ao tamanho e à largura das estrias; os valores foram superiores ao observado com a aplicação do laser de comprimento de onda $830 \mathrm{~nm}$.

\section{REFERÊNCIAS}

1. Wong R, Geyer S, Weninger W, Guimberteau J, Wong JK. The dynamic anatomy and patterning of skin. Exp Dermatol. 2016;25(2):92-8.

2. Dąbrowska AK, Rossi RM. The relationship between skin function, barrier properties, and body-dependent factors. Ski Res Technol. 2018;24(2):165-74.

3. Hague A, Hons M, Bayat A, Mbbs H, Eng M. Therapeutic targets in the management of striae distensae: a systematic review. J Am Dermatology. 2017;77(3):559-568.e18.

4. Al-Himdani S, Ud-Din S, Gilmore S, Bayat A. Striae distensae: a comprehensive review and evidence-based evaluation of prophylaxis and treatment. Br J Dermatol. 2014;170(3):527-47.

5. Liu L, Ma H, Li Y. Interventions for the treatment of stretch marks: a systematic review. Cutis. 2014;94(2):66-72.

6. Ud-Din S, Bayat A. New insights on keloids, hypertrophic scars, and striae. Dermatol Clin. 2014;32(2):193209.

7. Rezende PP, Pinheiro NM, Mendonça AC. Recursos terapêuticos para tratamento de estrias de distensão: uma revisão sistemática. JBCS. 2016;1(3):59-67.
8. Aldahan AS, Shah V V, Mlacker S, Samarkandy S, Alsaidan M, Nouri K. Laser and light treatments for striae distensae: a comprehensive review of the literature. Am J Clin Dermatol. 2016;17(3):239-56.

9. Elsaie ML, Baumann LS, Elsaaiee LT. Striae distensae (stretch marks) and different modalities of herapy: an update. Dermatologic Surg. 2009;35(4):563-73.

10. Leal CT, Bezerra AL, Lemos A. A efetividade do laser de HeNe 632,8 nm no reestabelecimento da integridade dos tecidos cutâneos em animais experimentais: revisão sistemática. Fisioter e Pesqui. 2012;19(3):291-6.

11. Leite SN, Masson-Meyers D dos S, Enwemeka CS, Leite1 SN, Andrade TAM de, Leite MN, et al. Phototherapy promotes healing of cutaneous wounds in undernourished rats. An Bras Dermatol. 2014;89(6):899-904.

12. Araújo M de JS, Martins GB. Utilização do diodo emissor de luz (LED) na cicatrização de queimaduras: revisão sistemática da literatura. Rev Pesq Fisio. 2019;9(1):1-12.

13. Silva MJS da, Tavares NJ, Röhl RCT, Portolez JLM, Fréz AR, Bertolini GRF. Efeitos do laser de baixa potência na remissão de feridas causadas pela psoríase vulgar. Rev Saúde e Pesqui. 2017;10(1):25-31.

14. McDaniel DH. Laser therapy of stretch marks. Dermatol Clin. 2002;20(1):67-76.

15. Busatta BB, Medeiro KC, Velozo LR, Kakihata CMM, Soares F dos S, Azevedo MRB, et al. Uso do laser de baixa potência em estrias de distensão: ensaio clínico randomizado controlado. Sci Med (Porto Alegre). 2018;28(2):ID28710.

16. Sardana K. Lasers for treating striae: an emergent need for better evidence. Indian J Dermatology, Venereol Leprol. 2014;80(5):392-5.

17. Castano AP, Dai T, Yaroslavsky I, Cohen R, Apruzzese WA, Smotrich MH, et al. Low-level laser therapy for zymosan-induced arthritis in rats: importance of illumination time. Lasers Surg Med. 2007;39(6):543-50.

18. Brassolatti P, de Andrade ALM, Bossini PS, Otterço 
AN, Parizotto NA. Evaluation of the low-level laser therapy application parameters for skin burn treatment in experimental model: a systematic review. Lasers Med Sci. 2018;33(5):1159-69.

19. Gál P, Stausholm MB, Kováč I, Dosedla E, Luczy J, Sabol F, et al. Should open excisions and sutured incisions be treated differently? A review and meta-analysis of animal wound models following low-level laser therapy. Lasers Med Sci. 2018;33(6):1351-62.

20. Maldaner DR, Azzolin VF, Barbisan F, Mastela MH, Teixeira CF, Dihel A, et al. In vitro effect of low-level laser therapy on the proliferative, apoptosis modulation, and oxi-inflammatory markers of premature-senescent hydrogen peroxide-induced dermal fibroblasts. Lasers Med Sci. 2019;In press.

21. Fridoni M, Kouhkheil R, Abdollhifar MA, Amini A, Ghatrehsamani M, Ghoreishi SK, et al. Improvement in infected wound healing in type 1 diabetic rat by the synergistic effect of photobiomodulation therapy and conditioned medium. J Cell Biochem. 2019;120(6):9906-16.

22. Lou Z, Zhang C, Gong T, Xue C, Scholp A, Jiang JJ. Wound-healing effects of 635-nm low-level laser therapy on primary human vocal fold epithelial cells: an in vitro study. Lasers Med Sci. 2018;33(3):469-77.

23. Freitas RP de A, Barcelos APM de, Nóbrega BM da, Macedo AB, Oliveira AR de, Ramos AM de O, et al. Laserterapia e microcorrente na cicatrização de queimadura em ratos. Terapias associadas ou isoladas? Fisioter e Pesqui. 2013;20(1):24-30.

24. Smith KC. Molecular targets for low level light therapy. Laser Ther. 2010;19(3):135-42.

25. Bjordal JM, Couppé C, Chow RT, Tunér J, Ljunggren EA. A systematic review of low level laser therapy with location-specific doses for pain from chronic joint disorders. Aust J Physiother. 2003;49(2):107-16.

Recebido em: 18/02/2019

Aceito em: 21/05/2019 